\title{
Conformal Array Pattern Synthesis Using a Hybrid WARP/2LB-MOPSO Algorithm
}

\author{
Roghieh Karimzadeh Baee, ${ }^{1}$ Keyvan Forooraghi, ${ }^{1}$ and Somayyeh Chamaani ${ }^{2}$ \\ ${ }^{1}$ The Electrical Engineering Department, Tarbiat Modares University, Jalal Ale Ahmad Highway, Tehran 14115-111, Iran \\ ${ }^{2}$ The Electrical and Computer Engineering Faculty, K. N. Toosi University of Technology, Tehran 1431714191, Iran \\ Correspondence should be addressed to Keyvan Forooraghi, keyvan_f@modares.ac.ir
}

Received 18 July 2012; Revised 31 August 2012; Accepted 4 September 2012

Academic Editor: Stefano Selleri

Copyright ( 2012 Roghieh Karimzadeh Baee et al. This is an open access article distributed under the Creative Commons Attribution License, which permits unrestricted use, distribution, and reproduction in any medium, provided the original work is properly cited.

\begin{abstract}
This paper addresses conformal array synthesis as a constrained multiobjective optimization problem. Simultaneous reduction of side lobe level (SLL) and cross-polarization (XPL) level is aimed with a constraint on main beam direction. A hybrid of weighted alternating reverse projection (WARP) and two local best multiobjective particle swarm optimization (2LB-MOPSO) is proposed to optimize the pattern. First, the WARP method finds a moderate and feasible solution. Second, 2LB-MOPSO begins with an initial population including the solution of WARP and penalty functions for constraint handling. Involving WARP result in the initial population of 2LB-MOPSO leads to higher convergence rate, avoiding local extermum traps and less sensitivity to penalty functions. Compared to WARP method which stagnates rapidly, the proposed hybrid method gives better SLL and XPL after adequate iterations. In addition, as 2LB-MOPSO offers a set of optimum solutions (Pareto front) instead of a single solution, this method provides more degrees of freedom in selection of proper practical arrays. Finally, to examine the mutual coupling consideration in array design, the same procedure was applied ignoring the mutual coupling between elements. The results show that the SLL and XPL strongly depend on mutual coupling.
\end{abstract}

\section{Introduction}

There is an increasing demand to conformal arrays because of their flexibility in attaching to prescribed shapes of vehicles and aircrafts or ships [1]. Among several conformal arrays, spherical arrays have received considerable attention because of their ability to provide single or multiple beams for full spherical coverage. These antennas have been proposed for applications such as ground station of satellite terminals and telemetry $[1,2]$. The main goal in these applications is to obtain pattern with low side lobe level (SLL) and crosspolarization level (XPL).

Unlike planar arrays, radiating elements in the conformal array are oriented in different directions and there is no closed form solution for analysis and consequently synthesis of these arrays [2].

If the array is conformed to curved surfaces, It is not possible to define an array factor. Therefore, unique challenges are posed in synthesis of these arrays. There are several methods to synthesize a desired pattern in conformal arrays [3-11]. Using high-speed computers, iterative methods are commonly used for pattern synthesis. The least mean squares (LMS) [5-7] and alternating projection [8] are extremely powerful for synthesizing the array pattern. The weighted LMS [9] was introduced where different weights for different directions are required. The weighted alternating reverse projection method (WARP) is similar to weighted LMS method. This method is a combination of weighting LMS and alternating projection. In our recent study, the WARP method has been used for synthesizing of hemispherical conformal array antenna [11]. The results show that WARP starts very well and generates appropriate results after a few iterations. However, it stagnates rapidly. On the contrary, there are evolutionary algorithms which search the solution space more efficiently but they may get trapped in local extremums. To get good results in a reasonable time and to avoid local extremum traps, we can mix the WARP method with an evolutionary algorithm. Some evolutionary 
algorithms such as genetic algorithms (GA) [12], simulated annealing [13], and particle swarm optimization (PSO) [14, 15] have been used in pattern synthesis. However, they are single objective techniques and do not consider different objectives (SLL and XPL) separately and hence no trade-off is possible. Therefore, multiobjective (Pareto) optimization techniques which find the best trade-off among conflicting objectives are preferred.

In this paper, a hemispherical conformal array antenna is simulated in CST. This array is composed of rectangular patches conformed to the hemispherical surface. The embedded pattern of each element are calculated by CST and then exported to a MATLAB program where an optimization routine based on hybrid of WARP and two local best multiobjective particle swarm optimization (2LB-MOPSO) is used to get the desired pattern with low SLL and XPL. To maintain main beam in a specific direction, a constraint is also added to the optimization which is handled by a constant penalty function. In addition to higher convergence rate and avoiding local extremum traps, involving WARP result in the initial population of 2LB-MOPSO leads to less sensitivity to penalty functions.

Compared to WARP method, the proposed hybrid method improves SLL and XPL about $3 \mathrm{~dB}$ and $3.5 \mathrm{~dB}$, respectively. To the best of our knowledge, it is the first time that the SLL and XPL are optimized where the embedded radiation pattern and the mutual coupling effect between elements are taken into account in the optimization process. Furthermore, this hybrid approach provides a set of optimum solutions instead of a single solution in a very short time. This set of solutions gives the designer more degrees of freedom for choosing the final array excitation.

\section{Pattern Formula of Conformal Array}

The farfield of an arbitrary array can be written in matrix form as follows [1, 9-11]:

$$
\mathbf{E}=\mathbf{Y A}
$$

where $\mathbf{E}$ is the directivity vector $\left[E\left(\theta_{1}, \varphi_{1}\right) \cdots E\left(\theta_{k}, \varphi_{k}\right)\right]^{T}$ in $k$ direction $\left(\left(\theta_{i}, \varphi_{i}\right), i=1,2, \ldots, k\right), \mathbf{A}$ is the excitation vector $\left[a_{1} \cdots a_{N}\right]^{T}$ of the array and $T$ denotes the transpose of a matrix. $\mathbf{Y}$ is the geometry-dependent matrix in spherical conformal arrays:

$$
Y_{i j}=g_{j}\left(\theta_{i}, \phi_{i}\right) \cdot e^{j k_{0}\left[\sin \left(\theta_{i}\right) \cos \left(\phi_{i}\right) x_{j}+\sin \left(\theta_{i}\right) \sin \left(\phi_{i}\right) y_{j}+\cos \left(\theta_{i}\right) z_{j}\right]},
$$

where $x_{j}, y_{j}$, and $z_{j}$ are positions of the $j$ th element and $g_{j}\left(\theta_{i}, \varphi_{i}\right)$ is field-gain of the $j$ th element in the direction of $\left(\theta_{i}, \varphi_{i}\right)$, that are not necessarily identical. E can be a $\theta$ or a $\varphi$ component and $\mathbf{Y}$ is different for different polarizations. Matrices $\mathbf{E}$ and $\mathbf{Y}$ can be written in both polarization components:

$$
\mathbf{E}=\left[\begin{array}{c}
\mathbf{E}_{\theta} \\
\mathbf{E}_{\phi}
\end{array}\right], \quad \mathbf{Y}=\left[\begin{array}{l}
\mathbf{Y}_{\theta} \\
\mathbf{Y}_{\phi}
\end{array}\right] .
$$

Therefore, both polarization components of farfield found from (1).
It can be shown [1] that if the mutual coupling of array is considered, then (1) must be modified to

$$
\mathbf{Y}_{\mathrm{s}}=\mathbf{Y}(\mathbf{I}+\mathbf{S})
$$

where $\mathbf{I}$ is the identity matrix and $\mathbf{S}$ denotes the $S$-parameter matrix.

In pattern synthesis of conformal array, we are looking for appropriate excitation vector to get the desired radiation pattern. The excitation values can be found by the least-mean squares (LMS) method minimizing the difference between the realized pattern and the desired pattern, respectively, $[1,3,4,9]$. In the weighted LMS method, excitation values can be calculated by introducing different weights for different directions [9]. Alternating projection method [16] introduces upper and lower boundaries in pattern and tries to hold the realized pattern within these boundaries. The weighted alternating reverse projection (WARP) method [10, 11] is a combination of weighting and modified alternating projection methods. As was shown in [12], the WARP method is applicable to synthesis of arbitrary array shapes and has better performance, compared to other methods.

\section{MOPSO Algorithm}

Pareto front in multiobjective contexts plays the same role as "extremum value" in single objective contexts. Since a single optimum solution is meaningless for multiobjective problems, Pareto front corresponds to a set of solutions which present the best trade-off among different objectives.

It is obvious that, the main aim of every multiobjective optimization algorithm is to find the Pareto optimal set. Traditional versions of Pareto optimization algorithms used to define a new aggregative weighted function from individual single objective functions [17]. Since these algorithms check the dominance criteria after each run of the new single objective problem, they tend to be very time-consuming. In addition, these classic approaches tend to be ineffective and inefficient due to their sensitivity to weighting factors [18]. More recent algorithms such as non-dominated sorting genetic algorithms II (NSGAII), multiobjective evolutionary algorithm (MOEA), and MOPSO omitted the "transform to single objective" stage and only use dominance criterion at each iteration. Among several dominance-based multiobjective algorithms, we applied the two lbest MOPSO (2LBMOPSO) [19] which has been recently introduced as a powerful multiobjective evolutionary algorithm and tested for several standard optimization problems. However, we also compared it with an earlier version of MOPSO proposed by Coello et al. [20] and have been applied for several electromagnetic optimization problems [21]. The results show superiority of 2LB-MOPSO in our array antenna synthesis.

\section{Results and Discussions}

The proposed method is applicable to arrays on arbitrary surfaces, but in this section the method is applied to synthesis of hemispherical array. Figure 1 shows the conformal array 


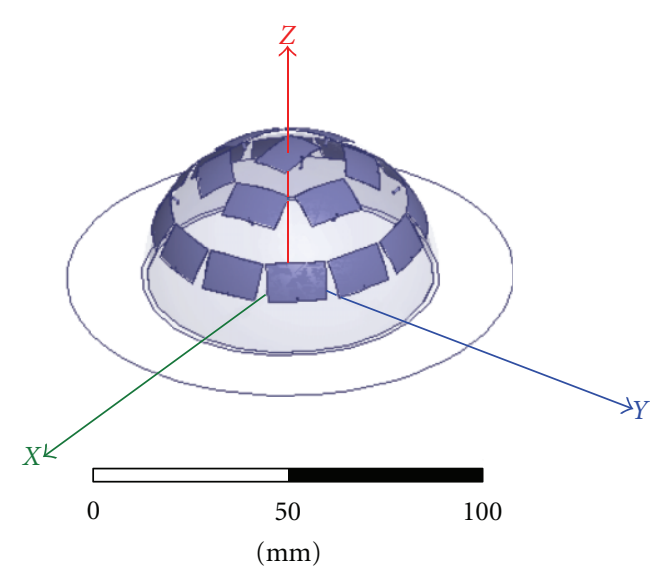

(a) 3D view

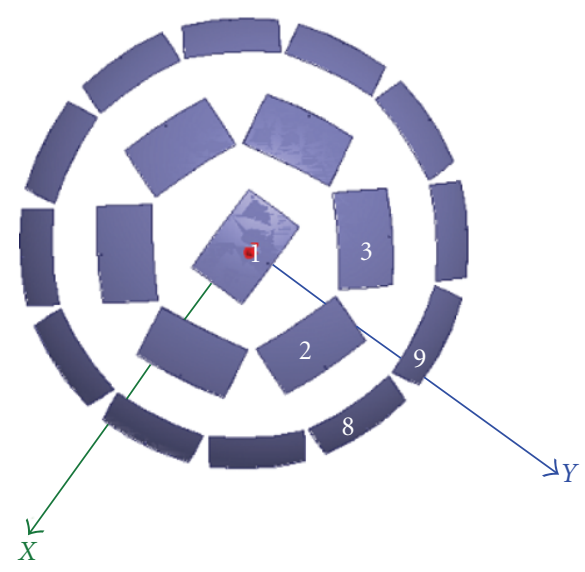

(b) Top view

FIgURE 1: Arrangement of a hemispherical conformal array antenna.

structure where the elements are numbered in counterclockwise sense in each row. This array consists of 19 rectangular patches wrapped around a hemisphere with a radius of $1.1 \lambda_{0}$, where $\lambda_{0}$ is the free space wavelength. The operating frequency is $8.3 \mathrm{GHz}$.

The dimensions of the patches are $10.5 \mathrm{~mm} \times 16 \mathrm{~mm}$. In this model, the patches are mounted on a substrate with thickness of $h=1.58 \mathrm{~mm}$ and the permittivity of $\varepsilon_{r 1}=2.52$. The $\theta$-polarized patches are fed through a coaxial probe. The Coaxial probe is centered in $\varphi$-direction, but its position is $4.5 \mathrm{~mm}$ far from the patch center in $\theta$ direction. The elements around the hemisphere are spaced apart by $0.58 \lambda_{0}$ in the first row from top of hemisphere and $0.5 \lambda_{0}$ in the second row. The rows are spaced apart by $0.58 \lambda_{0}$ from each other with different numbers of elements.

For simplicity, the azimuthal angle has been fixed and the elevation angle has been considered. The embedded-element patterns are evaluated by CST. For the embedded-element pattern extraction, only a single element is fed while the other elements are terminated in match loads. Therefore, they are being excited only by the mutually coupled fields. So, the embedded-pattern carries more information such as the effect of mutual coupling, surrounding elements, and a particular element grid $[1,11]$. The embedded-element pattern gives an indication of the performance of the fully excited array and serves as a valuable tool in the array design process.

The third definition of Ludwig is used to define the copolarization $E_{\text {copol }}$ and the cross-polarization $E_{\text {xpol }}[22,23]$ :

$$
\begin{gathered}
E_{\text {copol }}=E_{\theta} \cos \varphi-E_{\varphi} \sin \varphi, \\
E_{\text {xpol }}=E_{\theta} \sin \varphi+E_{\varphi} \cos \varphi .
\end{gathered}
$$

It follows from (5), $\left|E_{\phi}\right|$ and $\left|E_{\theta}\right|$ are the co- and the cross-polarization components in the $\varphi=90^{\circ}$ and $\varphi=270^{\circ}$ planes, respectively.

WARP and 2LB-MOPSO are applied to proposed hybrid method for optimization. Once the embedded pattern of elements are obtained, the remaining optimization procedure will be continued in MATLAB. First, the WARP method is applied to find a preliminary solution. In the second stage of our hybrid algorithm, 2LB-MOPSO searches the vicinity of WARP solution. The considered multiobjective problem is formulated as follows:

$$
\begin{aligned}
& \mathrm{XPL}=f_{1}=\max \left\{20 \log \left(\frac{E_{\mathrm{co}}\left(\theta_{\max }\right)}{E_{\mathrm{xpol}}(\theta)}\right)\right\}, \\
& \mathrm{SLL}=f_{2}=\max \left\{20 \log \left(\frac{E_{\mathrm{co}}\left(\theta_{\max }\right)}{E_{\mathrm{co}}\left(\theta_{S L_{i}}\right)}\right)\right\},
\end{aligned}
$$

Constraint: $\theta_{\max }=32$.

Since the pattern synthesis is not a convex problem, the rejection strategy cannot handle the constraint. Therefore, various penalty functions were tested. Generally, evolutionary algorithms are very sensitive to penalty functions and finding a proper penalty is a challenge [24]. However, in proposed hybrid method, adding a good feasible solution (WARP result) reduces this sensitivity whereas a constant penalty function in both SLL and XPL works properly.

In the hybrid method, Initial population of 2LB-MOPSO is random, except a particle that is the result of WARP. First, we compare the 2LB-MOPSO and MOPSO. For both cases the population size is NPOP $=50$ and the maximum number of generation is $T \max =1000$. Number of bins in both directions of objective space in for both 2LB-MOPSO and MOPSO is 20 and the in 2LB-MOPSO maximum count number which is checked to update lbests for each particle is 5. More details of algorithms can be found in $[19,20]$. As it is shown in Figure 2, the 2LB-MOPSO overcomes MOPSO. Therefore, the rest of optimizations in this paper are performed using 2LB-MOPSO.

In the following subsections, the proposed algorithm is applied for two case studies of hemispherical array synthesis considering and ignoring the mutual coupling effect.

4.1. Hemispherical Array Synthesis Considering Mutual Coupling Effect. In this section, the embedded pattern which has the information of the effect of mutual coupling has 


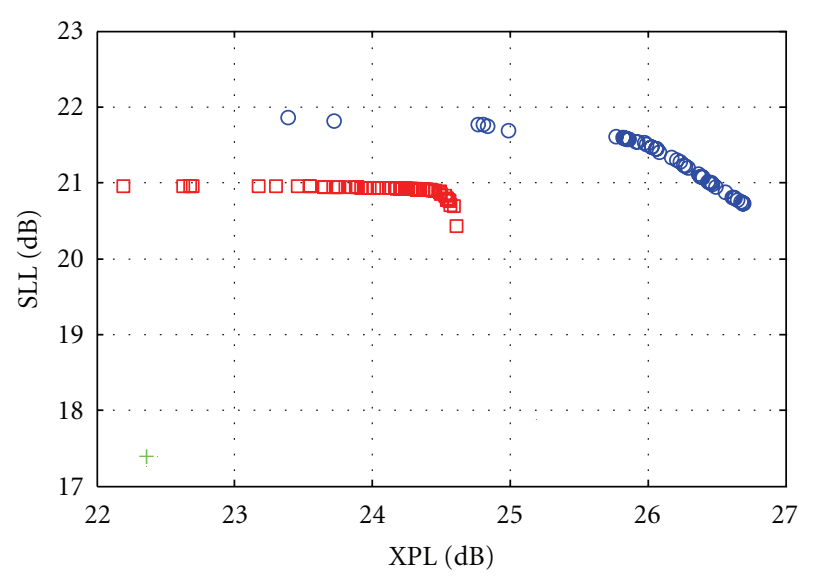

- 2 LB-MOPSO
+ MOPSO
+ WARP

FIGURE 2: Pareto fronts obtained by the 2LB-MOPSO and MOPSO.

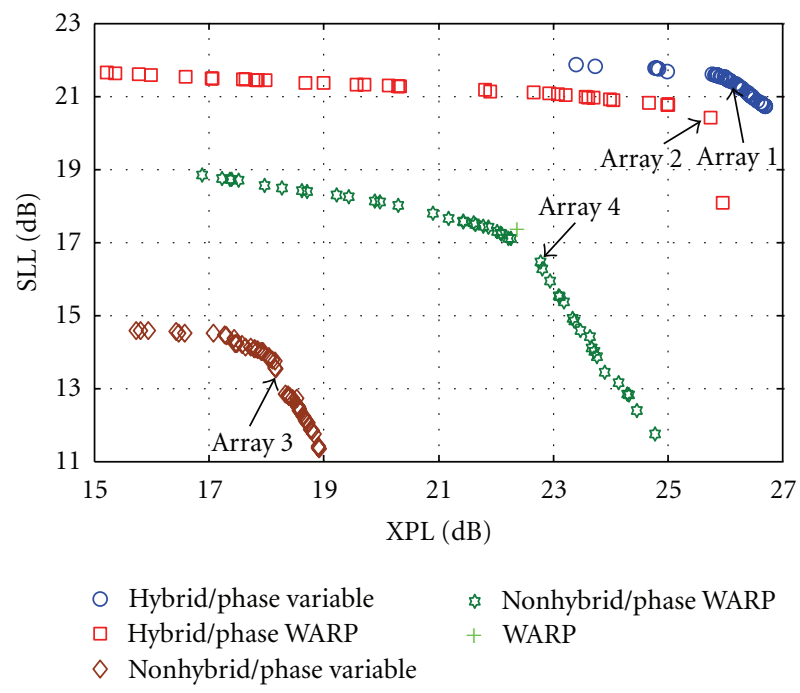

Figure 3: Resulted Pareto front of hybrid and nonhybrid, variable phase and WARP phase compared to WARP method result.

been used. To investigate the effectiveness of proposed hybrid method, the hybrid and nonhybrid 2LB-MOPSO were compared. In both of hybrid and nonhybrid scenarios, excitation phase of each elements can be fixed (equal to WARP result phase) or tunable. When phase of excitation is equal to the WARP result phase, the number of variables would be half.

Figure 3 shows Pareto front for 4 cases as follows.

Hybrid/Variable Phase. Uses the amplitude and phases of the WARP method in the initial population and both phase and amplitude of excitations are tunable.
TABLE 1: Comparison of SLL and XPL.

\begin{tabular}{lcc}
\hline Sample & SLL $(\mathrm{dB})$ & XPL $(\mathrm{dB})$ \\
\hline WARP & 17.5 & 22.5 \\
Array 1 & 21.5 & 26 \\
Array 2 & 20.5 & 25.7 \\
Array 3 & 13.5 & 18.2 \\
Array 4 & 16.45 & 22.8 \\
\hline
\end{tabular}

Nonhybrid/Variable Phase. Does not use WARP solution as an experience and both phase and amplitude of excitations take participates in the optimization.

Hybrid/WARP Phase. Excitation phase of each element is equal to the excitation phase of finding in WARP for total iteration and only amplitudes are optimized. It uses the amplitude and phase of the WARP method in the initial population.

Nonhybrid/WARP Phase. Phase and amplitude of elements are changed during the optimization. It does not use amplitude of WARP result in the initial solution.

As shown in Figure 3, hybrid methods dominate nonhybrid methods. In addition, tuning the phase provides more degrees of freedom and consequently better results. So the best Pareto belongs to hybrid/variable phase method and the worst Pareto belongs to nonhybrid/WARP phase. As a result, If WARP solution uses as an experience, this helps 2LBMOPSO to obtain better element excitation which results in lower SLL and XPL. Furthermore, only nonhybrid/WARP phase is dominated by WARP result. If nonhybrid method is applied, WARP phase can find better results in comparison with variable phase. To have a numerical comparison, 4 different samples from Pareto fronts are selected (Array 1Array 4). Corresponding SLL and XPLs are listed in Table 1. From Table 1 obviously Array 1 has about $3 \mathrm{~dB}$ and $3.5 \mathrm{~dB}$ SLL and XPL, respectively, better than WARP.

Figure 4 compares the co- and cross-polarization patterns obtained by WARP with one of the hybrid and nonhybrid solutions (indicated by Array 1 and Array 3 in Figure 3). As shown in Figure 4, beam width changes in nonhybrid/variable phase and Array 3 solution has higher SLL and XPL than Array 1 and WARP.

The comparison between the proposed hybrid method radiation patterns and the full wave CST simulation for Array 1 is shown in Figure 5 which proves a good agreement between these two results.

4.2. Hemispherical Array Synthesis Neglecting Mutual Coupling Effect. In this section, the mutual couplings between elements are neglected. The farfield of each element are exported from CST with assuming that they are isolated. Then WARP and the proposed hybrid method are applied to this array. Figure 6 shows the resulted Pareto front. It can be seen that the proposed hybrid method at most improves SLL and XPL around $1.5 \mathrm{~dB}$ and $0.5 \mathrm{~dB}$, respectively, in comparison with WARP in this situation. 


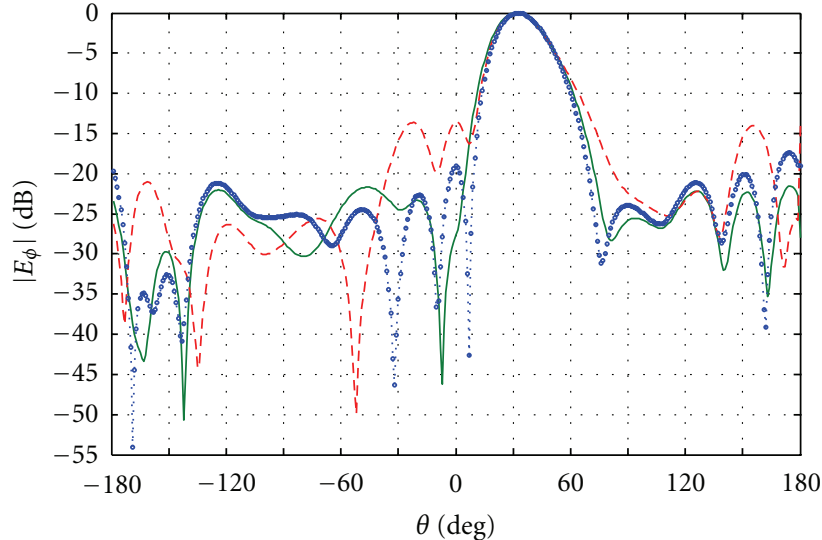

- Array 1

..... WARP

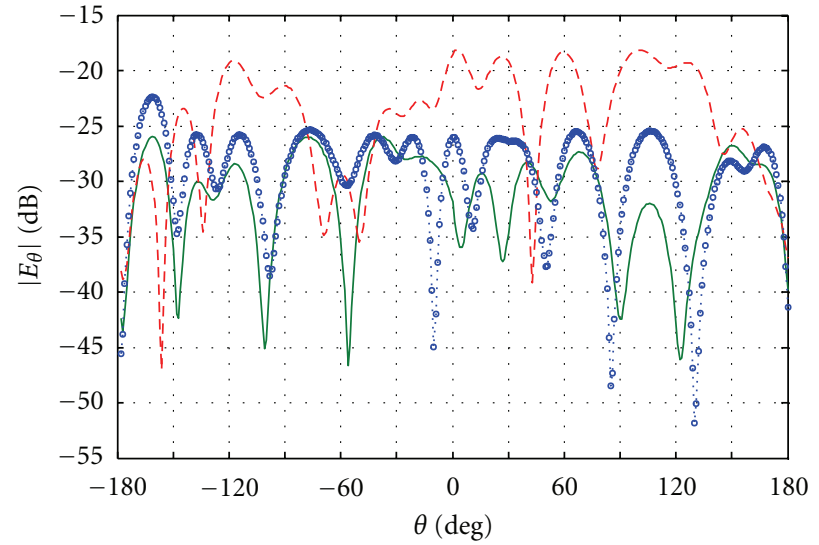

- Array 1

- - Array 3

.... WARP

(a) $E_{\phi}$

(b) $E_{\theta}$

FIGURE 4: Comparison of the realized pattern using hybrid, nonhybrid, and WARP methods in the planes $\varphi=90$ and $\varphi=270$.

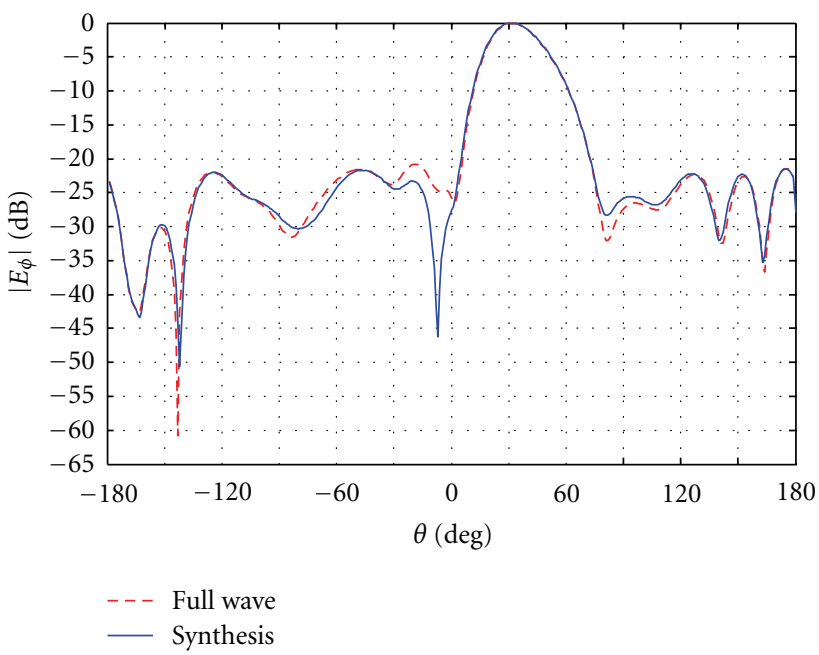

(a) $E_{\phi}$

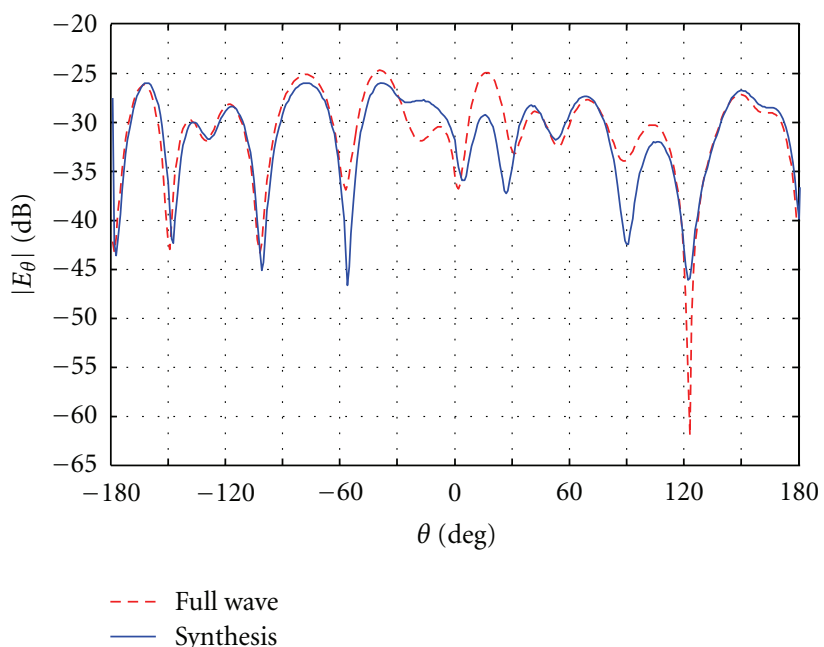

(b) $E_{\theta}$

FIGURE 5: A comparison between the realized pattern using the proposed hybrid method and the simulated by CST in the planes $\varphi=90$ and $\varphi=270$.

The co- and cross-polarization patterns, one of the hybrid solutions (indicated by Array 5 in Figure 6) that were obtained by MATLAB and full wave, were compared in Figure 7. As it illustrated that the SLL and XPL of full wave simulation changed in comparison with MATLAB result, because the mutual coupling effect in synthesizing of array was not considered in synthesizing by MATLAB.

Also, the mutual coupling in conformal array may be less than planner array, but SLL and XPL are strongly dependent on mutual coupling. Therefore, the mutual coupling plays an important role in synthesis. It is essential to include these effects in the synthesis of array such as conformal arrays.

\section{Conclusion}

A novel hybrid of 2LB-MOPSO and WARP was proposed and used to optimize the pattern of a hemisphericalconformal-phased array antenna. In the case of known geometry distribution of array elements, the excitation amplitude, and phase of each element were optimized to reduce SLL and XPL, simultaneously.

The results show that the hybrid method leads to better pattern compared to both WARP and 2LB-MOPSO (nonhybrid). In addition, this multiobjective algorithm provides a set of optimum solutions instead of a single solution which helps the designer in his/her final selection. The result of 


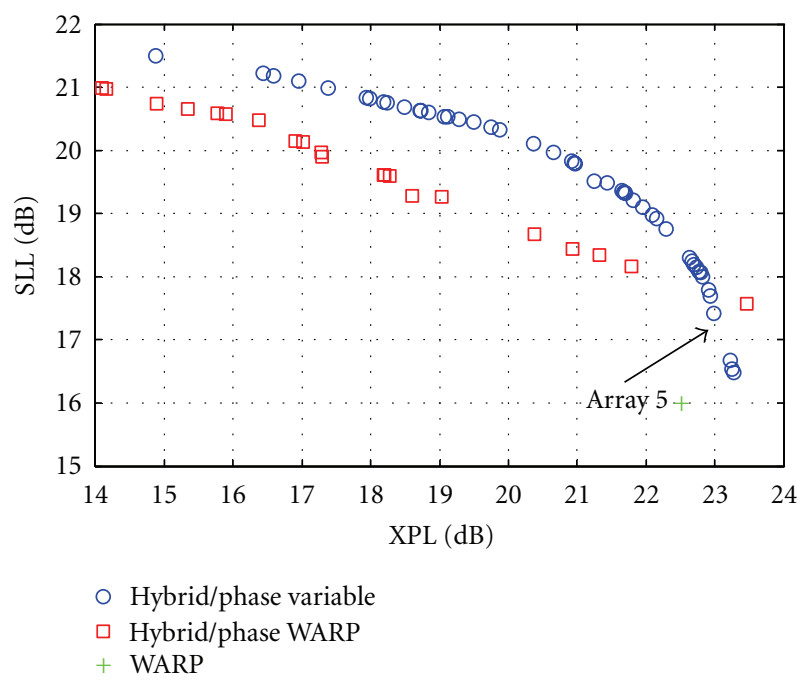

FIGURE 6: Resulted Pareto front of hybrid variable phase compared to WARP with assuming isolated elements.

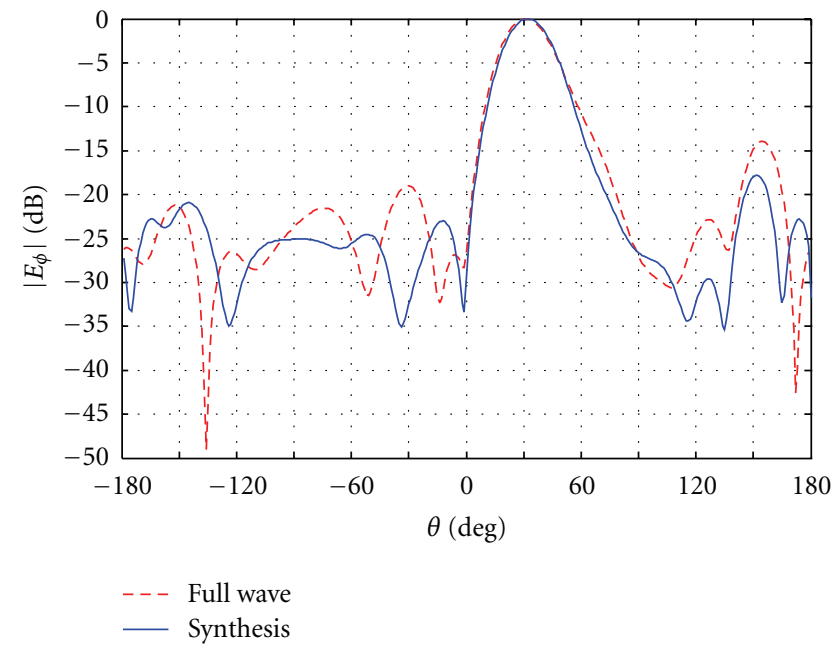

(a) $E_{\phi}$

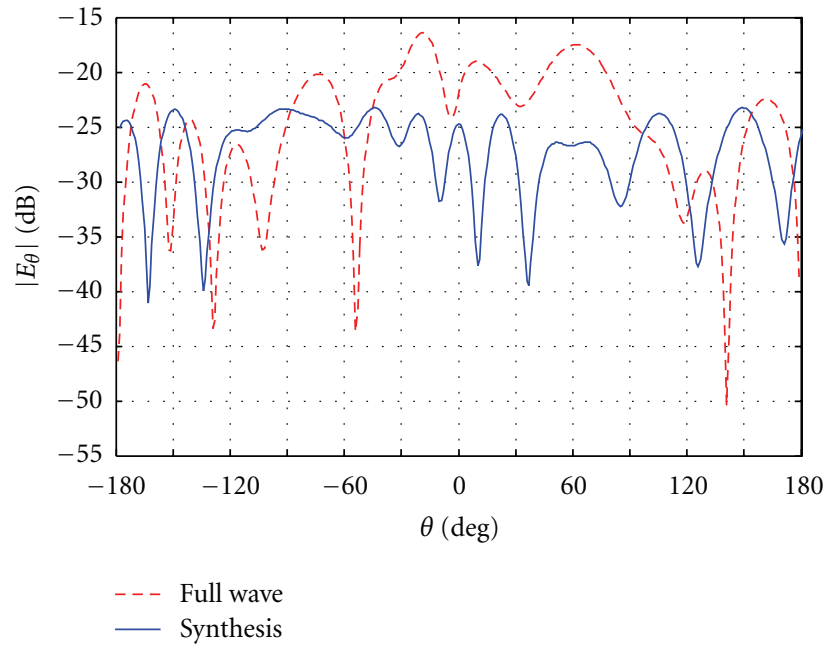

(b) $E_{\theta}$

FIGURE 7: A comparison of the realized pattern using the proposed hybrid method and the simulated by CST without considering of the mutual coupling in the planes $\varphi=90$ and $\varphi=270$.

the proposed method was verified by full wave simulations. Comparing the results of MATLAB codes and full wave simulations, shows the effect of mutual coupling in the synthesis of hemispherical conformal array should not be neglected because the SLL and XPL are strongly dependent on mutual coupling.

\section{Acknowledgments}

The paper is written in the loving memory of late Professor Mohammad Hakkak, who had passed away during this research and his useful help will be remembered forever. The authors also would like to thank the Research Institute of ICT (former Iran Telecommunication Research Center) for its financial support.

\section{References}

[1] L. Josefsson and P. Persson, Conformal Array Antenna, Theory and Design, Wiley-IEEE Press, New Jersey, NJ, USA, 2006.

[2] C. Dohmen, J. W. Odendaal, and J. Joubert, "Synthesis of conformal arrays with optimized polarization," IEEE Transactions on Antennas and Propagation, vol. 55, no. 10, pp. 2922-2925, 2007.

[3] B. Tomasic, J. Turtle, and S. Liu, "A geodesic sphere phased array for satellite control and communication," in Proceedings of the 18th International Conference on Applied Electromagnetics and Communications (ICECOM '05), pp. 487-493, Dubrovnik, Croatia, 2005.

[4] S. Rupcic, V. Mandric, and D. Zagar, "Reduction of sidelobes by nonuniform elements spacing of a spherical antenna array," Radioengineering, vol. 20, no. 1, pp. 299-306, 2011. 
[5] B. D. Carlson and D. Willner, "Antenna Pattern synthesis using weighted least squares," in Proceedings of the Institute of Electrical and Electronics Engineers (IEEE '92), vol. 139, pp. 1116, 1992.

[6] L. I. Vaskelainen, "Iterative least-squares synthesis methods for conformal array antennas with optimized polarization and frequency properties," IEEE Transactions on Antennas and PropagationNo, vol. 48, 6, pp. 987-991, 2000.

[7] P. N. Fletcher and M. Dean, "Least squares pattern synthesis for conformal arrays," Electronics Letters, vol. 34, no. 25, pp. 2363-2365, 1998.

[8] O. M. Bucci, G. D'Elia, and G. Romito, "Power synthesis of conformal arrays by a generalised projection method," IEE Microwaves, Antennas and Propagation, vol. 142, no. 6, pp. 467-471, 1995.

[9] L. I. Vaskelainen, "Iterative least-squares synthesis methods for conformai array antennas with optimized polarization and frequency properties," IEEE Transactions on Antennas and Propagation, vol. 45, no. 7, pp. 1179-1185, 1997.

[10] A. Haddadi, A. Ghorbani, and J. Rashed-Mohassel, "Cosecant squared pattern synthesis using a weighted alternating reverse projection method," IET Microwaves, Antennas and Propagation, vol. 5, pp. 1789-1795, 2011.

[11] R. Karimzadeh, M. Hakkak, A. Haddadi, and K. Forooraghi, "Conformal array pattern synthesis using the warp method considering mutual coupling and embedded-element pattern effects," IET Microwaves, Antennas and Propagation, vol. 6, pp. 621-626, 2012.

[12] D. Marcano and F. Durán, "Synthesis of antenna arrays using genetic algorithms," IEEE Antennas and Propagation Magazine, vol. 42, no. 3, pp. 12-20, 2000.

[13] N. H. Farhat and B. Bai, "Phased-array antenna pattern synthesis by simulated annealing," Proceedings of the Institute of Electrical and Electronics Engineers (IEEE '87), vol. 75, no. 6, pp. 842-844, 1987.

[14] D. W. Boeringer and D. H. Werner, "Efficiency-constrained particle swarm optimization of a modified Bernstein polynomial for conformal array excitation amplitude synthesis," IEEE Transactions on Antennas and Propagation, vol. 53, no. 8, pp. 2662-2673, 2005.

[15] A. A. Khan and A. K. Brown, "Difference pattern synthesis using a particle swarm optimizer," in Proceedings of the Loughborough Antennas and Propagation Conference (LAPC '09), pp. 329-332, November 2009.

[16] O. M. Bucci, G. Franceschetti, G. Mazzarella, and G. Panariello, "General projection approach to array synthesis," in Proceedings of the International Symposium Digest: Antennas and Propagation, pp. 146-149, June 1989.

[17] J. Lei, G. Fu, L. Yang, and D. M. Fu, "Multi-objective optimization design of the Yagi-Uda antenna with an Xshape driven dipole," Journal of Electromagnetic Waves and Applications, vol. 21, no. 7, pp. 963-972, 2007.

[18] A. P. Engelbrecht, Fundamentals of Computational Swarm Intelligence, John Wiley \& Sons, 2005.

[19] S. Z. Zhao and P. N. Suganthan, "Two-lbests based multiobjective particle swarm optimizer," Engineering Optimization, vol. 43, no. 1, pp. 1-17, 2011.

[20] C. A. Coello, G. T. Pulido, and M. S. Lechuga, "Handling multiple objectives with particle swarm optimization," IEEE Transactions on Evolutionary Computation, vol. 8, no. 3, pp. 256-279, 2004.
[21] S. Chamaani, S. A. Mirtaheri, M. Teshnehlab, M. A. Shoorehdeli, and V. Seydi, "Modified Multi-objective Particle Swarm Optimization for electromagnetic absorber design," Progress in Electromagnetics Research, vol. 79, pp. 353-366, 2008.

[22] K. F. Lee and K. M. Luk, Microstrip Patch Antennas, Imperial College Press, 2011.

[23] A. C. Ludwig, "The definition of cross polarization," IEEE Transactions on Antennas and Propagation, vol. AP-21, pp. 116-119, 1973.

[24] B. Y. Qu and P. N. Suganthan, "Constrained multi-objective optimization algorithm with an ensemble of constraint handling methods," Engineering Optimization, vol. 43, no. 4, pp. 403-416, 2011. 

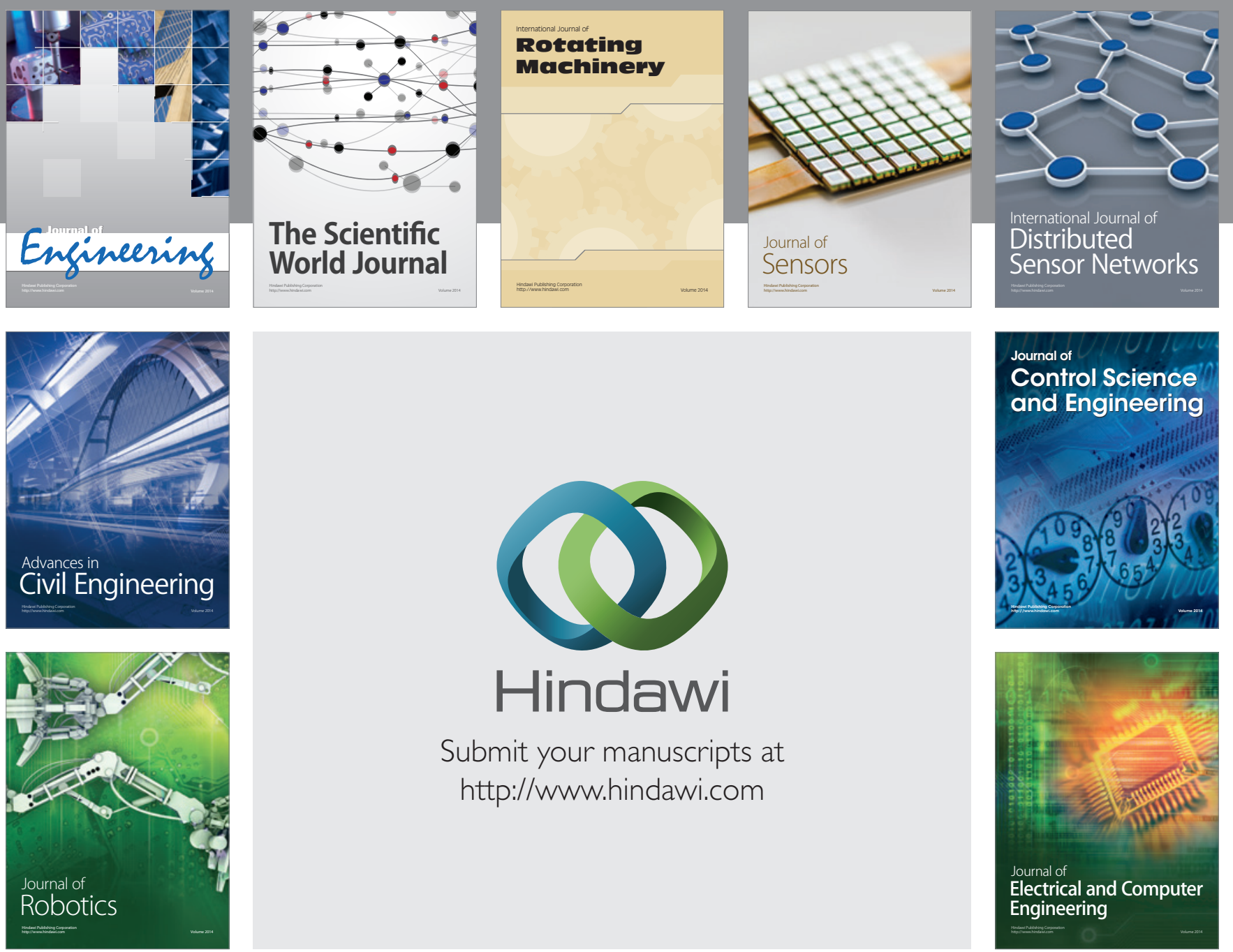

Submit your manuscripts at

http://www.hindawi.com
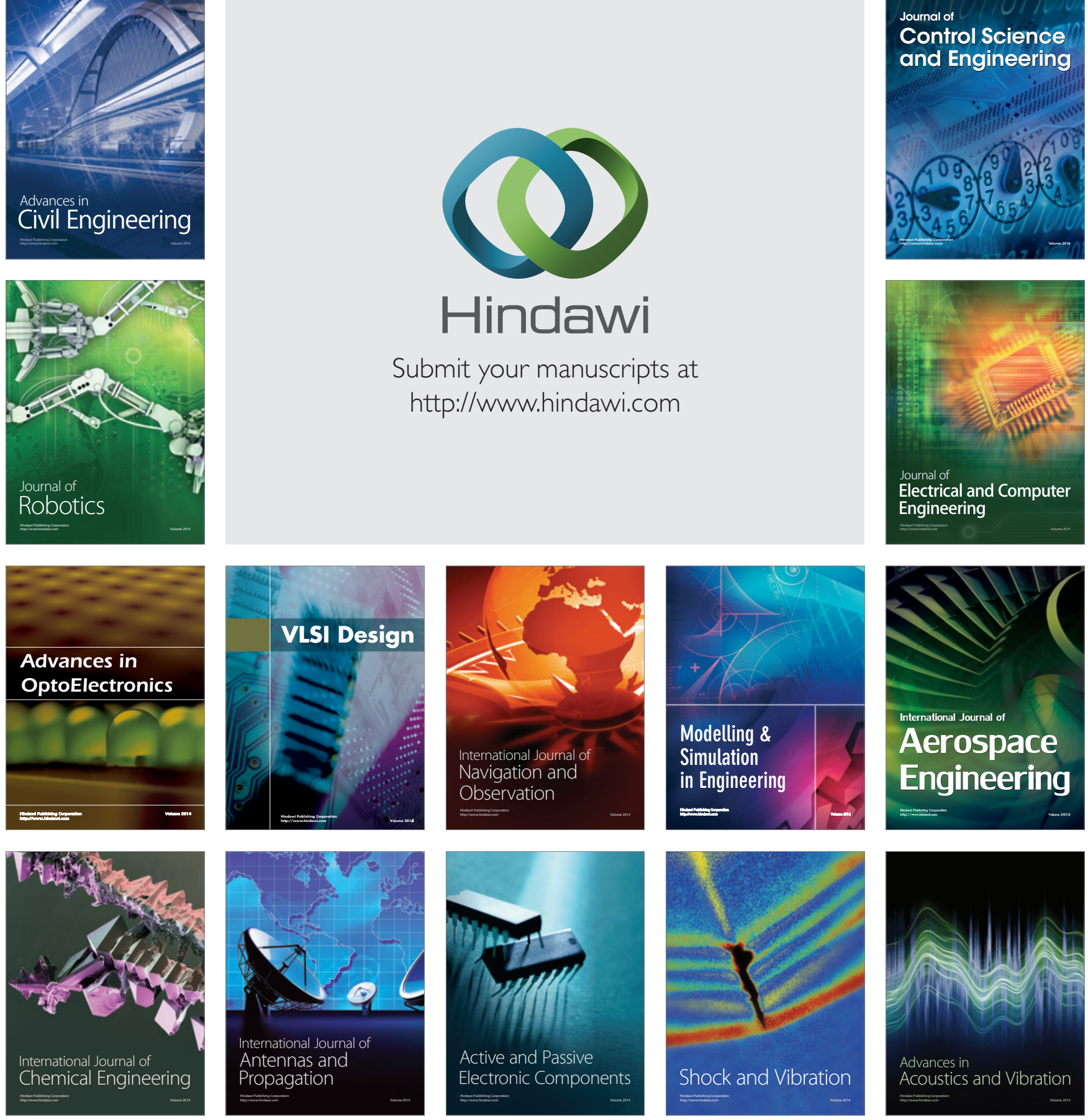